\title{
Well-quasi-order of relabel functions
}

\author{
Jean Daligault ${ }^{1}$ Michael Rao ${ }^{1}$ Stéphan Thomassé ${ }^{1}$ \\ LIRMM-Université Montpellier II, 161 rue Ada, 34392 Montpellier Cedex, France \\ daligault@lirmm.fr \\ rao@lirmm.fr \\ thomasse@lirmm.fr
}

\begin{abstract}
We define $\mathrm{NLC}_{k}^{\mathcal{F}}$ to be the restriction of the class of graphs $\mathrm{NLC}_{k}$, where relabelling functions are exclusively taken from a set $\mathcal{F}$ of functions from $\{1, \ldots, k\}$ into $\{1, \ldots, k\}$. We characterize the sets of functions $\mathcal{F}$ for which $\mathrm{NLC}_{k}^{\mathcal{F}}$ is well-quasiordered by the induced subgraph relation $\leq_{i}$. Precisely, these sets $\mathcal{F}$ are those which satisfy that for every $f, g \in \mathcal{F}$, we have $\operatorname{Im}(f \circ g)=\operatorname{Im}(f)$ or $\operatorname{Im}(g \circ f)=\operatorname{Im}(g)$. To obtain this, we show that words (or trees) on $\mathcal{F}$ are well-quasi-ordered by a relation slightly more constrained than the usual subword (or subtree) relation.

A class of graphs is $n$-well-quasi-ordered if the collection of its vertex-labellings into $n$ colors forms a well-quasi-order under $\leq_{i}$, where $\leq_{i}$ respects labels. Pouzet [15] conjectured that a 2-well-quasi-ordered class closed under induced subgraph is in fact $n$-well-quasi-ordered for every $n$. A possible approach would be to characterize the 2-well-quasi-ordered classes of graphs. In this respect, we conjecture that such a class is always included in some well-quasi-ordered $\mathrm{NLC}_{k}^{\mathcal{F}}$ for some family $\mathcal{F}$. This would imply Pouzet's conjecture.
\end{abstract}

\section{Introduction}

Let $S$ be a set and $\leq$ be a quasi-order on $S$, i.e. a reflexive and transitive relation. Given a sequence $\left(x_{i}\right)_{i \in \omega}$ of elements of $S$, a good pair consists of two elements $x_{i} \leq x_{j}$, with $i<j$. A sequence with no good pair is called a bad sequence of $(S, \leq)$. A quasi-order with no bad sequence is a well-quasi-order.

There are other equivalent presentations of the notion of well-quasi-ordering (see for instance [12]). A quasi-order is a well-quasi-order if and only if it

1 Research supported by the french ANR-project "Graph decompositions and algorithms (GRAAL)"

Preprint submitted to Elsevier

19 March 2008 
has no infinite antichain and no infinite strictly decreasing sequence. Also, a quasi-order is a well-quasi-order if and only if every infinite sequence has an infinite increasing subsequence. Equivalently, a quasi-order is a well-quasiorder if and only if every nonempty subset of $S$ has a nonempty finite set of minimal elements (up to equivalence).

The theory of well-quasi-ordering has been flourishing. Higman's theorem states that the set of words over a well-quasi-ordered set is well-quasi-ordered by the subword relation [7], and this has been extended by Kruskal's to trees [11]. Robertson and Seymour's celebrated graph minor theorem [16] asserts that the minor relation is a well-quasi-order on the set of finite graphs. It implies that every graph class closed under minor (or minor ideal) can be characterized by a finite list of excluded minors. This in turn implies that every minor ideal can be recognized in polynomial time.

The class of finite graphs is not well-quasi-ordered by the induced subgraph relation since the cycles form an antichain. The good properties of the minor ideals ensured by the minor theorem do not hold for induced subgraph ideals (for instance, the set of paths, which is well-quasi-ordered does not have a finite set of forbidden induced subgraphs). This is a motivation to the stronger notion of 2-well-quasi-ordering.

In the following, we will be exclusively interested in the induced subgraph relation. Throughout this paper, we will abbreviate "well-quasi-ordered by the induced subgraph relation" in well-quasi-ordered, being understood that we are dealing with the induced subgraph relation.

An extension of the notion of well-quasi-order is the notion of $n$-well-quasiorder (see Kriz and Thomas [9] for a more general discussion in terms of QOcategory). A set $S$ of graphs is $n$-well-quasi-ordered if the class $\hat{S}$ consisting of all vertex $n$-coloring of graphs in $S$, is well-quasi-ordered by $\leq_{1}$, where $G \leq_{1} G^{\prime}$ if there is an injection from $V$ to $V^{\prime}$ preserving adjacency and color. The set $S$ is $\infty$-well-quasi-ordered if $S$ is $n$-well-quasi-ordered, for any $n \geq 1$. Being 2-well-quasi-ordered is a stronger property than being well-quasi-ordered, for instance the set of paths is not 2-well-quasi-ordered.

The notion of 2-well-quasi-ordering is especially interesting in view of algorithmic properties, as induced subgraph ideals which are 2-well-quasi-ordered can be characterized by a finite list of forbidden induced subgraphs, and thus are polynomially recognizable. Our ultimate aim would be to characterize the 2-well-quasi-ordered ideals of graphs, in order to prove the following conjecture of Pouzet [15], also appearing in Fraïssé [4]:

Conjecture 1 An induced subgraph ideal is 2-well-quasi-ordered if and only if it is $\infty$-well-quasi-ordered. 


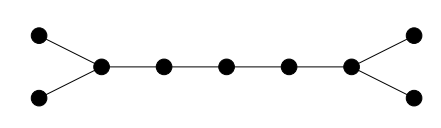

Fig. 1. The graph $G_{5}$

We will come back to this topic in Section 5. Our main purpose here is to study a restriction of the hierarchy of graph classes NLC.

The class $\mathrm{NLC}_{k}$ of $k$-node labelled controlled graphs was introduced in [18]. Let $\mathcal{F}$ be a set of functions from $\{1, \ldots, k\}$ into $\{1, \ldots, k\}$. The class $\mathrm{NLC}_{k}^{\mathcal{F}}$ is defined recursively by using $k$ node-labels and three operators: $\bullet_{i}, \circ_{f}$ and $\chi_{S}$. For $i \in\{1, \ldots, k\}$, the operator $\bullet_{i}$ creates a single vertex labelled by $i$. The operator $\circ_{f}$, with $f \in \mathcal{F}$, applied to a labelled graph replaces every label $i$ with $f(i)$. The operator $\chi_{S}$, with $S \subseteq\{1, \ldots, k\} \times\{1, \ldots, k\}$, applied to two labelled graphs $G$ and $H$ in this order, creates the disjoint union of graphs $G$ and $H$, and for all $(i, j) \in S$ adds edges between every vertex of label $i$ in $G$ and every vertex of label $j$ in $H$. The class $\mathrm{NLC}_{k}$ is defined to be $\mathrm{NLC}_{k}^{\Phi}$ where $\Phi$ is the set of all functions from $\{1, \ldots, k\}$ into $\{1, \ldots, k\}$. The $N L C$-width of $G$ is the minimum $k$ for which some labelling of $G$ is in $\mathrm{NLC}_{k}$.

It is not known whether there exists a polynomial time algorithm computing a NLC decomposition using $k$ colors for graphs in $\mathrm{NLC}_{k}$. Only the cases $k=1$ (which corresponds to cographs) and $k=2$ (see [8]) have been solved so far. Computing the NLC-width is NP-hard (see [6]).

The NLC-width has a strong link with another well-known parameter: the clique-width, introduced by Courcelle et al. (see [1]). NLC-width and cliquewidth indeed differ by a factor at most 2 (more precisely, the clique-width of a graph is bounded below by its NLC-width, and above by twice its NLC-width). Moreover, transformations respecting these bounds between decompositions of the two types can be done in linear time.

The class of graphs $\mathrm{NLC}_{1}$ is well-quasi-ordered, see [2] and [17] for the countable case. The class of graphs $\mathrm{NLC}_{2}$ is well-quasi-ordered (and even $\infty$-wellquasi-ordered), this easily follows from the results in [8]. Indeed, the $\mathrm{NLC}_{2}$ prime graphs for the modular decomposition are constructible in $\mathrm{NLC}_{2}$ without relabelling, and thus form a well-quasi-ordered family by Kruskal's tree theorem. However, the class $\mathrm{NLC}_{3}$ is not well-quasi-ordered, as it contains for every $i$ the graph $G_{i}$ (a path of length $i$ with two pending vertices added to each extremity) depicted in Fig. 1. These graphs indeed do not form a well-quasi-ordered family. Allowing all relabelling operators $o_{f}$ is too much to construct a well-quasi-ordered class of graphs if we have at least 3 colors. This is why we define a restriction of NLC, using only relabelling operators from a specified set of functions $\mathcal{F}$. Our main purpose is to characterize the sets $\mathcal{F}$ such that $\mathrm{NLC}_{k}^{\mathcal{F}}$ is well-quasi-ordered. We will see that $\mathrm{NLC}_{k}^{\mathcal{F}}$ is well-quasiordered (equivalently $\infty$-well-quasi-ordered) if and only if it does not contain 
arbitrarily large paths.

In Section 2, we introduce a binary relation $\preceq$ on set of functions. In Section 3 we introduce a subword order $\leq$ on words labelled with a set of functions which is more constrained that Higman's order. In Section 4 we extend $\leq$ to trees, with the purpose of applications to $\mathrm{NLC}_{k}^{\mathcal{F}}$ expressions. In Section 5, we characterize the sets $\mathcal{F}$ for which $\mathrm{NLC}_{k}^{\mathcal{F}}$ is well-quasi-ordered. In the final section, we discuss Pouzet's conjecture on $n$-well-quasi-ordering.

Throughout this paper, we will obtain the following equivalent characterizations of $\mathcal{F}$ :

- The set $\mathcal{F}$ is totally quasi-ordered by $\preceq$.

- The set of words on $\mathcal{F}$ is well-quasi-ordered by $\leq$.

- The set of trees on $\mathcal{F}$ is well-quasi-ordered by $\leq$.

- The set of graphs $\mathrm{NLC}_{k}^{\mathcal{F}}$ is well-quasi-ordered.

- The set of graphs $\mathrm{NLC}_{k}^{\mathcal{F}}$ is $\infty$-well-quasi-ordered.

- The set of graphs $\mathrm{NLC}_{k}^{\mathcal{F}}$ does not contain arbitrarily large paths.

\section{Totally ordered sets of functions}

Let $\mathcal{F}$ be a set of functions from $\{1, \ldots, k\}$ into $\{1, \ldots, k\}$ closed under composition (with the convention that the identity function $\varepsilon$ belongs to $\mathcal{F}$ ). The key-definition of this section is the following. Let us say that $f \preceq g$ whenever $\operatorname{Im}(f \circ g)=\operatorname{Im}(f)$.

Assume that $\preceq$ is total on $\mathcal{F}$, i.e. for every $f, g$ in $\mathcal{F}$, at least one of $f \preceq g$ and $g \preceq f$ holds. This implies in particular that $\operatorname{Im}\left(f^{2}\right)=\operatorname{Im}(f)$ for all $f \in \mathcal{F}$. Observe that $f \preceq g$ implies that $|\operatorname{Im}(f)| \leq|\operatorname{Im}(g)|$.

Lemma 1 If $\preceq$ is total on $\mathcal{F}$, then $\preceq$ is transitive.

Thus $\preceq$ is a reflexive and transitive relation, in other words $\preceq$ is a total quasiorder on $\mathcal{F}$. This is equivalent to the existence of a partition of $\mathcal{F}$ into $t$ equivalence classes $F_{1}, \ldots, F_{t}$ such that $f \in F_{i}$ and $g \in F_{j}$ verify $f \preceq g$ if and only if $i \leq j$.

Lemma 2 When $\mathcal{F}$ is totally quasi-ordered by $\preceq$, the equivalence classes $F_{1}, \ldots, F_{t}$ are exactly the classes of functions having an image of the same size, in increasing order of the image size.

Observe that the top class $F_{t}$ contains $\varepsilon$, and contains only permutations.

Lemma 3 For all $i, F_{i}$ and $\cup_{k \geq i} F_{k}$ are closed under composition. 
Lemma 4 The functions of the bottom class $F_{1}$ verify a "left-cancellation" identity:

$$
\forall f \in F_{1}, \forall h, h^{\prime} \in \mathcal{F}, \text { if } h \circ f \circ h^{\prime}=h \circ f \text { then } f \circ h^{\prime}=f
$$

Here is an example of a set of functions which is totally ordered by $\preceq$. An $(i, j)$-cast, with $i \leq j$, is a function $f$ from $\{1, \ldots, k\}$ into itself such that $f(l)=l$ for all $l<i$ and $f(l)=j$ whenever $i \leq l$. It is routine to check that the set of casts is indeed totally ordered by $\preceq$. We feel that the following problem would give some insight on the well-quasi-ordered $\mathrm{NLC}_{k}^{\mathcal{F}}$ classes:

Problem 2 Find a generic class of functions $\mathcal{G}$ (like casts for instance) such that for every totally ordered $\mathcal{F}$ and $k$, there exists some $k^{\prime}$ for which $N L C_{k}^{\mathcal{F}}$ is included in $N L C_{k^{\prime}}^{\mathcal{G}}$

Such a class of function would describe much more precisely how to construct the well-quasi-ordered classes $N L C_{k}^{\mathcal{F}}$.

\section{Words on functions}

An $\mathcal{F}$-word is a finite word on the alphabet $\mathcal{F}$, i.e. a finite sequence $f_{1}, \ldots, f_{l}$ of elements of $\mathcal{F}$. Let $\mathcal{W}^{\mathcal{F}}$ be the set of $\mathcal{F}$-words. Let $M=f_{1}, \ldots, f_{l}$ and $M^{\prime}=f_{1}^{\prime}, \ldots, f_{l^{\prime}}^{\prime}$ be two $\mathcal{F}$-words. The word $M$ is a subword of $M^{\prime}$ if there exists an increasing injection $\phi$ from $\{1, \ldots, l\}$ into $\left\{1, \ldots, l^{\prime}\right\}$ such that $f_{i}=f_{\phi(i)}^{\prime}$. Higman's theorem asserts that the subword partial order is a well-quasi-order when the alphabet is finite. In our more constrained partial order on $\mathcal{F}$-words, we have $M \leq M^{\prime}$ if two conditions are satisfied:

- There is a function $\phi$ for which $M$ is a subword of $M^{\prime}$.

- For all $1 \leq i<l$, we have $f_{i}=f_{\phi(i)}^{\prime} \circ f_{\phi(i)+1}^{\prime} \circ \cdots \circ f_{\phi(i+1)-1}^{\prime}$.

Thus, when $M \leq M^{\prime}$ and $i<j$, the composition of functions $f_{i} \circ f_{i+1} \circ \cdots \circ f_{j-1}$ is equal to the function $f_{\phi(i)}^{\prime} \circ f_{\phi(i)+1}^{\prime} \circ \cdots \circ f_{\phi(j)-1}^{\prime}$. And since $f_{j}=f_{\phi(j)}^{\prime}$, we also have $f_{i} \circ f_{i+1} \circ \cdots \circ f_{j}=f_{\phi(i)}^{\prime} \circ f_{\phi(i)+1}^{\prime} \circ \cdots \circ f_{\phi(j)}^{\prime}$.

Our goal is to prove here that $\mathcal{W}^{\mathcal{F}}$ is well-quasi-ordered by $\leq$ if and only if $\preceq$ is total on $\mathcal{F}$. For this, we have to be a little bit more general and need to consider $\mathcal{W}_{Q}^{\mathcal{F}}$, the set of words on the set $\mathcal{F} \times Q$, where $Q$ is a set endowed by a well-quasi-ordered $\leq_{Q}$.

We naturally extend the partial order $\leq$ on $\mathcal{W}_{Q}^{\mathcal{F}}$. For $w$ in $\mathcal{W}_{Q}^{\mathcal{F}}$ and $1 \leq x \leq|w|$ we denote by $\left(f_{x}^{w}, q_{x}^{w}\right)$ the $x^{\text {th }}$ letter of $w$. For any couple of indices $a, b$, with $1 \leq$ $a<b \leq|w|$, we define $L^{w}(a, b)$ to be the composition $f_{a}^{w} \circ f_{a+1}^{w} \circ \ldots \circ f_{b-1}^{w}$. When 
$a=b$, we set $L^{w}(a, b)=\epsilon$. Let $\phi$ be an increasing injection from $\{1, \ldots,|w|\}$ into $\left\{1, \ldots,\left|w^{\prime}\right|\right\}$. We say that $\phi$ is compatible with labels if $f_{x}^{w}=f_{\phi(x)}^{w^{\prime}}$ and $q_{x}^{w} \leq_{Q} q_{\phi(x)}^{w^{\prime}}$. We say that $\phi$ preserves path-composition if for every $x<|w|$, we have that $L^{w}(x, x+1)=L^{w^{\prime}}(\phi(x), \phi(x+1))$ (observe that by definition we have $\left.L^{w}(x, x+1)=f_{x}^{w}\right)$. We write $w \leq w^{\prime}$ if there exists an increasing injection $\phi$ from $\{1, \ldots,|w|\}$ into $\left\{1, \ldots,\left|w^{\prime}\right|\right\}$ compatible with labels and preserving path-composition. When $\phi$ is only compatible with labels, we simply say that $w$ is a subword of $w^{\prime}$ and write $w \leq_{0} w^{\prime}$.

Theorem 1 The set of words $\mathcal{W}_{Q}^{\mathcal{F}}$ is well-quasi-ordered by $\leq$ if and only if $\preceq$ is a total quasi-order on $\mathcal{F}$.

\section{Trees on functions}

We extend in this section our results to trees. However, since the arguments are similar to the previous section, we will not give the same level of details, especially concerning the verification of path-composition.

A structured tree is a finite tree where the childs of a node are ordered from left to right. Our trees have their nodes labelled by a well-quasi-ordered set $Q$. We denote by $\mathcal{T}_{Q}^{\mathcal{F}}$ the set of structured rooted trees with nodes labelled by $\mathcal{F} \times Q$, where $\mathcal{F}$ is as usual a set of functions. A node $x$ is then labelled by a pair $l(x)=(f(x), q(x))$. We simply write $\mathcal{T}^{\mathcal{F}}$ when there is no additional label $Q$. The set of nodes of $T$ is denoted by $V(T)$. We write $x \wedge y$ for the least common ancestor of $x$ and $y$. We say that $(x, y)$ is an $\operatorname{arc}$ of $T$ when $x$ is the father of $y$. A sequence of nodes $z_{0}, z_{1}, \ldots, z_{n}$ is a downward path in $T$ if $\left(z_{i}, z_{i+1}\right)$ is an arc, for every $i=0, \ldots, n-1$. For such a downward path $z_{0}, z_{1}, \ldots, z_{n}$, we denote by $L\left(z_{0}, z_{n}\right)$ the composition $f\left(z_{0}\right) \circ f\left(z_{1}\right) \circ \ldots \circ f\left(z_{n-1}\right)$.

Let us define a partial order $\leq$ on $\mathcal{T}_{Q}^{\mathcal{F}}$ which extends the order $\leq$ on words. Precisely, let us write that $T \leq T^{\prime}$ if there exists an injection $\phi$ from $V(T)$ into $V\left(T^{\prime}\right)$ such that:

- $\phi$ preserves descendance.

- $\phi$ preserves least common ancestors, i.e. $\phi(x \wedge y)=\phi(x) \wedge \phi(y)$.

- $\phi$ preserves left/right order, i.e. if $x$ and $y$ are not in descendance relation, and the branch of $x \wedge y$ containing $x$ is to the left of the one containing $y$, the same holds for the branches of $\phi(x) \wedge \phi(y)$ containing $\phi(x)$ and $\phi(y)$.

- $\phi$ preserves labels, i.e. $f(x)=f(\phi(x))$ and $q(x) \leq_{Q} q(\phi(x))$.

- $\phi$ preserves path-composition if for any $\operatorname{arc}(x, y)$ in $T$, we have that $L(x, y)=$ $L(\phi(x), \phi(y))$, i.e. $f(x)=L(\phi(x), \phi(y))$.

When $\phi$ satisfies all these properties except possibly path-composition, we 
simply write $T \leq_{0} T^{\prime}$. Kruskal's tree theorem asserts that $\leq_{0}$ is a well-quasiorder on the set of trees.

This more constrained order relation $\leq$ presents some analogies with the socalled gap-condition embedding studied by Kriz in [10]. For instance, when the class of functions $\mathcal{F}$ is totally ordered, and hence partitioned into $F_{1}, \ldots, F_{t}$, the path-composition property implies that if $y$ is a child of $x$ and $f(x)$ belongs to $F_{i}$, then every fonction of the product $L(\phi(x), \phi(y))$ belong to classes with height at least $i$. It could be interesting to state a common generalization of these results, possibly involving ordinal functions.

Theorem $2 \mathcal{T}_{Q}^{\mathcal{F}}$ is well-quasi-ordered by $\leq$ if and only if $\preceq$ is total on $\mathcal{F}$.

\section{$5 \quad$ NLC with restricted relabelling functions}

We can see $\mathrm{NLC}_{k}^{\mathcal{F}}$ expressions as binary trees, where the leaves are labelled by $\boldsymbol{\bullet}_{i}$, the nodes of degree 1 by ${ }_{f}$, and the nodes of degree 2 by $\chi_{S}$. To fit in the framework of the previous section, we add an extra label to every node of such an $\mathrm{NLC}_{k}^{\mathcal{F}}$ construction tree, to see $\chi_{S}$ and $\bullet_{i}$ as identity relabelling functions. For this, replace $\bullet_{i}$ with $\left(\varepsilon, \bullet_{i}\right)$, of with $\left(f, \circ_{f}\right)$ and $\chi_{S}$ with $\left(\varepsilon, \chi_{S}\right)$. Such a tree is a construction tree for the vertex-colored graph corresponding to this NLC $_{k}^{\mathcal{F}}$ expression. Let $T_{G}$ be a construction tree for a vertex-colored graph $G$. Let $x$ be a vertex of $G$ with color $i$ which corresponds to the leaf $x^{\prime}$ of $T_{G}$ and $y$ be an ancestor of $x^{\prime}$ in $T_{G}$. When we apply the operation corresponding to the node $y$ of $T_{G}$ to the vertex $x$, the color of $x$, denoted by $c_{x}(y)$ is exactly $L\left(y, x^{\prime}\right)(i)$.

Lemma 5 Let $G$ and $H$ be two vertex $k$-colored graphs together with their $N L C_{k}^{\mathcal{F}}$ construction trees $T_{G}$ and $T_{H}$. If $T_{G} \leq T_{H}$, then $G \leq_{i} H$.

Proof: Let $\phi$ be an injection from $V\left(T_{G}\right)$ into $V\left(T_{H}\right)$. The restriction of $\phi$ on the leaves of $T_{G}$ can be seen as an injection from $V(G)$ into $V(H)$. Let $x, y$ be two vertices of $G$, with $x$ on the left of $y$ in $T$. Then $x$ and $y$ are neighbours in $G$ if and only if their least common ancestor in $V\left(T_{G}\right)$ is a node labelled by $\chi_{S}$ with $\left(c_{x}(x \wedge y), c_{y}(x \wedge y)\right) \in S$. As $\phi$ preserves labels, path composition and right/left order, this is the case if and only if $\phi(x)$ and $\phi(y)$ are neighbours in $H$. So $G \leq_{i} H$.

Theorem 2 immediately gives:

Corollary 1 If $\preceq$ is total on $\mathcal{F}$, then $N L C_{k}^{\mathcal{F}}$ is well-quasi-ordered by $\leq_{i}$.

Moreover, since we can always add some extra vertex-labels, we obtain that NLC $_{k}^{\mathcal{F}}$ is $\infty$-well-quasi-ordered when $\preceq$ is total on $\mathcal{F}$. The converse of the 
previous corollary actually holds:

Theorem $3 N L C_{k}^{\mathcal{F}}$ is well-quasi-ordered by $\leq_{i}$ if and only if $\preceq$ is total on $\mathcal{F}$.

Proof: Assume that $\preceq$ is not total on $\mathcal{F}$, and let $(f, g)$ be an incomparable pair for the relation $\preceq$. Let us show that for any $n \geq 1$, the graph $G_{n}$ depicted in Fig. 1 is in $\operatorname{NLC}_{k}^{\mathcal{F}}$. The set $\left\{G_{n} \mid n \in \omega\right\}$ is clearly not well-quasi-ordered.

Assume first that $f=g$, that is $\left|\operatorname{Im}\left(f^{2}\right)\right|<|\operatorname{Im}(f)|$. Hence there exist $x, y$ $\notin \operatorname{Im}\left(f^{2}\right)$, such that $f(x)=y$. To construct $G_{n}$, start from two vertices labelled by $y$ and one vertex labelled by $x$, and apply $\chi_{\{(x, y)\}}$ to form a path of length 2. Relabel by $f$. Observe that the two extremities of this path will never be again labelled by $x$ or $y$ since their labels will stay within $\operatorname{Im}\left(f^{2}\right)$. Add a vertex labelled by $x$, apply again $\chi_{\{(x, y)\}}$. This adds an edge between the middle vertex of the path and the new one. Then relabel by $f$, and keep on building the path up to the desired length. The point is that after any step, the extremity of the path is distinguished by its label from the other vertices. When the last vertex of the path has been added (with label $x$ as usual), add two isolated vertices with label $x$ for instance, and apply $\chi_{\{(x, x)\}}$, completing the graph $G_{n}$.

We can generalize this when $f$ and $g$ are distinct. An $f$-class is a subset $S$ of $\{1, \ldots, k\}$ such that $|f(S)|=1$ and which is maximum with respect to inclusion. Since $f \npreceq g$, there exists an $f$-class disjoint from $\operatorname{Im}(g)$. Let $x$ be one of its elements. Similarly, let $y$ be in a $g$-class disjoint from $\operatorname{Im}(f)$. Let us prove by induction that for every $n$, we can build paths of length $2 n$ where the last vertex is labelled by $y$ and the other vertices are labelled in the set $\operatorname{Im}(f)$. We will therefore be able to build graphs $G_{n}$ for arbitrarily large $n$ then, adding two pending nodes on each extremity as in the previous case.

To start with, take a vertex $z \in \operatorname{Im}(f)$, add a vertex $y$, and apply $\chi_{\{(z, y)\}}$. Now assume that we have a path of length $2 n$ where the last vertex is labelled by $y$ and the other vertices by some elements of $\operatorname{Im}(f)$. Relabel by $g$. Observe that the last vertex is still distinguished from the rest. Add a vertex $x$. At this point, no other vertex has label $x$, since $x$ is not in $\operatorname{Im}(g)$. Apply $\chi_{\{(x, y)\}}$. This constructs a path of length $2 n+1$. Now relabel by $f$, add a vertex $y$ and apply $\chi_{\{(y, x)\}}$ in order to get a path of length $2(n+1)$ which satisfies the induction hypothesis.

To sum-up the different results of this section, let us mention the following equivalent statements:

- $\mathrm{NLC}_{k}^{\mathcal{F}}$ is well-quasi-ordered by $\leq_{i}$

- $\mathrm{NLC}_{k}^{\mathcal{F}}$ is $\infty$-well-quasi-ordered by $\leq_{i}$

- $\preceq$ is total on $\mathcal{F}$

- $\mathrm{NLC}_{k}^{\mathcal{F}}$ does not contain arbitrarily large paths. 


\section{$6 \infty$-well-quasi-ordered classes of graphs}

As we have mentioned before, one important motivation for the notion of 2-well-quasi-ordered class is that it can be described by a finite set of bounds.

Proposition 3 Let $S$ be a 2-well-quasi-ordered induced subgraph ideal. There are finitely many graphs in the complement $\bar{S}$ of $S$ which are minimal with respect to the induced subgraph relation.

Proof: By contradiction, we assume that the border (the set of minimal graphs in $\bar{S}) B$ is infinite. For every graph $G$ in $B$, choose a vertex, color its neighbours red and its non-neighbours black, and delete it. Call the resulting graph $G^{\prime}$. The set $B^{\prime}=\left\{G^{\prime} \mid G \in B\right\}$ is infinite, and we have that $B^{\prime} \subseteq S$, by minimality of the border graphs. Thus there exist two graphs $G_{1}^{\prime}$ and $G_{2}^{\prime}$ in $B^{\prime}$, such that $G_{1}^{\prime} \leq_{1} G_{2}^{\prime}$. Hence $G_{1}$ is an induced subgraph of $G_{2}$, contradicting the fact that $G_{2}$ is in $B$.

This implies that any 2-well-quasi-ordered induced subgraph ideal is polynomially recognizable. This means in particular that for a set $\mathcal{F}$ totally quasiordered by $\preceq$, the class $\mathrm{NLC}_{k}^{\mathcal{F}}$ is polynomially recognizable.

The following question would give an answer to Pouzet's conjecture.

Conjecture 4 If $\mathcal{G}$ be a 2-well-quasi-ordered induced subgraph ideal, there exists a well-quasi-ordered set $N L C_{k}^{\mathcal{F}}$ which contains $\mathcal{G}$.

We have no clue concerning this problem. One first step would be to show that if a class of graph has unbounded clique-width, then it is not 2-well-quasiordered. The next step would be to show that if indeed a subclass of $N L C_{k}$ is 2-well-quasi-ordered, then it is contained in some well-quasi-ordered set $\mathrm{NLC}_{k^{\prime}}^{\mathcal{F}}$. Finally, let us mention a question which would push Pouzet's conjecture to a full extent. The answer for cographs can be found in [17].

A quasi-order $Q$ is a better-quasi-order if the class of countable ordinals labelled by $Q$ is a wqo.

Conjecture 5 Let $\mathcal{G}$ be a class of countable graphs. If the class of finite induced subgraphs $\mathcal{G}_{F}$ of $\mathcal{G}$ is 2-well-quasi-ordered, then $\mathcal{G}$ is better-quasi-ordered for every better-quasi-ordered vertex-label. 


\section{References}

[1] B. Courcelle, J. Engelfriet, G. Rozenberg, Handle-Rewriting Hypergraph Grammars, J. Comput. Syst. Sci., 46 (1993), 218-270.

[2] P. Damaschke, Induced subgraphs and well-quasi-ordering, J. Graph Theory, 14 (1990), 427-435.

[3] G. Ding, Subgraphs and well-quasi-ordering, J. Graph Theory, 16 (1992), 489502.

[4] R. Fraïssé, Theory of Relations, North Holland, Studies in logic, 118 (1986).

[5] F. Gurski, Characterizations for restricted graphs of NLC-width 2, Theoretical Computer Science, 372 (2007), 108-114.

[6] F. Gurski, E. Wanke, Minimizing NLC-width is NP-complete (Extended Abstract), in: Proc. Graph-Theoretical Concepts in Computer Science, Lecture Notes in Computer Science, Vol. 3787, Springer, Berlin, (2005), 69-80.

[7] G. Higman, Ordering by divisibility in abstract algebras, Proc. London Math. Soc., 2 (1952), 326-336 .

[8] O. Johansson, NLC2-Decomposition in Polynomial Time, International Journal of Foundations of Computer Science, 11 (2000), 373-395.

[9] I. Kriz, R. Thomas, On well-quasi-ordering finite structures with labels, Graphs Combin., 6 (1990), 41-49.

[10] I. Kriz, Well-Quasiordering Finite Trees with Gap-Condition. Proof of Harvey Friedman's Conjecture, Annals of Math., 130 (1989), 215-226.

[11] J. B. Kruskal, Well-quasi-ordering, the tree theorem, and Vazsonyi's conjecture, Transactions of the American Mathematical Society, 95 (1960), 210-225.

[12] J. B. Kruskal, The theory of well-quasi-ordering, a frequently rediscovered concept, Journal of Combinatorial Theory, Series A, 13 (1972), 297-305.

[13] C. St. J. A. Nash-Williams, On well-quasi-ordering infinite trees, Proc. Cambr. Philos. Soc., 61 (1965), 697-720.

[14] M. Petkovsek, Letter graphs and well-quasi-order by induced subgraphs, Discrete Math., 244 (2002), 375-388.

[15] M.Pouzet, Un bel ordre d'abritement et ses rapports avec les bornes d'une multirelation, C. R. Acad. Sci. Paris Sér. A-B, 274 (1972), 1677-1680.

[16] N. Robertson, P. Seymour, Graph Minors. XX. Wagner's conjecture, Journal of Combinatorial Theory, Series B, 92 (2004), 325-357.

[17] S. Thomassé, On better-quasi-ordering countable series-parallel orders, Trans. Amer. Math. Soc., 352 (2000), 2491-2505.

[18] E. Wanke, $k$-NLC Graphs and Polynomial Algorithms, Discrete Applied Mathematics, 54 (1994), 251-266. 